\title{
Determination of total protein content in biomedical products by the PDMS-assisted lab-in-a-syringe assay using 3D printed scaffolds removal
}

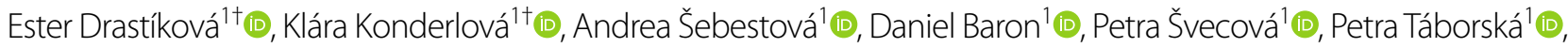

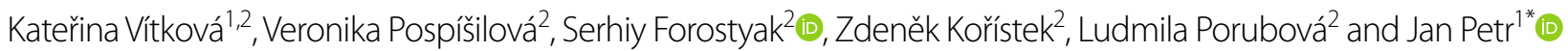

\begin{abstract}
The aim of our work was to develop a low-cost, portable device for the fast and easy determination of total protein content by using PDMS-based lab-in-a-syringe technology with removal of 3D-printed channels. We proposed two designs with a one-step PDMS curing and a two-step PDMS-curing fabrication procedure. The one-step PDMS microdevices were found to be the best in the view of preparation, repeatability, and stability of the reagent. This design was then applied for the determination of total protein content in biomedical products using the Bradford assay.
\end{abstract}

Keywords: Lab-in-a-syringe, 3D printing, Microfluidics, PDMS, Total protein content

\section{Introduction}

The development of simple, low-cost, and efficient methods and/or devices for bioanalysis is tremendously valuable for many application fields, including self-testing in medicine, non-professional assays for determination of food quality, and portable environmental testing. The concept of low-cost microfluidics, introduced in 2007 (Martinez et al. 2007), fits the needs for such devices. They are made from cheap materials, like paper or wax, with cheap procedures, like common printing, drawing by pencils, or ironing. These devices can be used for many purposes, as recently reviewed (Carrell et al. 2019; Salentijn et al. 2018; Akyazi et al. 2018; Weng et al. 2019). Moreover, the concept of paper-based microfluidics was broadening out by the combination of these devices with

\footnotetext{
*Correspondence: jan.petr@upol.cz

†Ester Drastíková and Klára Konderlová have contributed equally to this work

${ }^{1}$ Department of Analytical Chemistry, Faculty of Science, Palacký University Olomouc, 17. Listopadu 12, 77146 Olomouc, Czech Republic Full list of author information is available at the end of the article
}

other devices and techniques known from "classical" microfluidics. There are articles combining paper-based devices with foil or PDMS, including "classical" stereolithography procedures (Kamali et al. 2018; Montgomery et al. 2018; Maskova et al. 2017).

Interestingly, in 2015, the lab-in-a-syringe (LIS) assay in microfluidics based on the lateral flow assay in a holder connected to the syringe was introduced (Pauli et al. 2015). This work was then followed by Zor and Bekar (Zor and Bekar 2017) for discrimination of enantiomers. In 2017, Hárendarčíková et al. (Hárendarčíková et al. 2017) developed the assay where the reaction and detection pads were introduced directly into the syringe barrel. However, the concept of lab-in-a-syringe or labin-syringe (or in-syringe) analysis is much older. Generally, it was used in many research fields including solid phase synthesis in organic chemistry (Cankařová et al. 2019; Schütznerová et al. 2018), for microextraction (Maya et al. 2012; Yu et al. 2019; Tang et al. 2019; Šrámková et al. 2018; Barreto et al. 2019), or for focusing of analytes before mass spectrometry in analytical chemistry (Mikhail et al. 2019). 
Among the many applications of these low-cost devices, there is a need for fast, cheap, and portable devices that determine total protein content to control the production of biopharmaceuticals. Generally, these biomedical products or biopharmaceutical products are drugs manufactured (or semi-synthesized) from biological sources such as cells, cellular components, or cell cultures. During the production process, especially in the development stages, there is a need for cheap and fast control of cell growth and drug production (Lomonossoff and D'Aoust 2016; Parr et al. 2016; Hidalgo et al. 2018; Torano et al. 2019). Typically, total protein content assays are used to show the increase in protein production. They are, of course, not so precise in comparison with proteomics, but they are enough to check the production process. Moreover, the total protein content assay is also used as the first and cheap test in the quality control of prepared biopharmaceuticals. If the batch passes such control, it can be characterized by means of more specialized, and more expensive, techniques. As can be seen from the praxis as well as publications, the assay is still considered as the "golden standard" in any proteinbased application (Radosevich and Burnouf 2010; Raynal et al. 2014). Traditionally, a total protein content assay is based on the colorimetric reaction of samples with a specific reagent, e.g., Bradford (Bradford 1976). This is done in laboratory conditions that need a transfer of samples from the biotechnology production plant. In this view, the use of a portable and low-sample consumption assay would make all the processes easier.

Consequently, the aim of our work was to develop a low-cost, portable device for the fast and easy determination of total protein content. We decided to use the lab-in-a-syringe technology as the ideal platform for such a task. However, the lab-in-a-syringe assays (Hárendarčíková et al. 2017) use a large sample in the milliliter range volumes that is not favorable for production control. To decrease the amount of sample required, the assay should be inserted into the smaller syringe, but this limits the user-friendliness of the analysis. The second possibility is to form channels in the syringe barrel. Here, the combination of 3D printing and PDMS-based microfluidics can be used. This 3D printing approach is adopted in microfluidics, as reviewed in other works (Hartings and Ahmed 2019; Manzanares Palenzuela and Pumera 2018; Dixit et al. 2018; Bhattacharjee et al. 2016; $\mathrm{Au}$ et al. 2016; Li et al. 2019a; Gong et al. 2018; Salentijn et al. 2017). Most of the papers address the direct 3D printing of materials and formation of final microfluidic devices (MacDonald et al. 2017; Li et al. 2017a, 2017b, 2019b; Bhattacharjee et al. 2018; Vittayarukskul and Lee 2017; Romanov et al. 2018; Beauchamp et al. 2019). Interestingly, there is a second approach where $3 \mathrm{D}$ printed structures are placed in PDMS and removed after PDMS curing. This approach was presented for the first time in two pioneering works in 2015. Hwang et al. (Hwang et al. 2015) mechanically removed the scaffolds by rotating the molds, and Saggiomo and Velders (Saggiomo and Velders 2015) created so called ESCARGOT methodology where the ABS polymer scaffolds are printed, inserted into PDMS, and then removed with acetone. These approaches were recently followed by Dahlberg et al. (2018) who used a water-soluble polyvinyl alcohol (PVA) filament for rapid production of PDMS microfluidic devices. These approaches seem to be ideal for our work because they do not need high investment costs either.

\section{Experimental \\ Chemicals and materials}

The Bradford reagent, bovine serum albumin (BSA), phosphoric acid, sodium hydroxide, dimethylglyoxime, nickel (II) chloride hexahydrate, potassium bromide, and ammonium hydroxide were bought from SigmaAldrich (St. Louis, MO, USA), all at p.a. quality. Deionized water of $18.2 \mathrm{M} \Omega . \mathrm{cm}$ was prepared by the MilliQ system from Millipore (Burlington, MA, USA). The prepolymer of polydimethylsiloxane (PDMS) and the curing agent known as Sylgard 184 were purchased from Elchemco (Prague, Czech Republic). Materials, mainly $2 \mathrm{~mL}$ syringes, were bought from Chirana (Stará Turá, Slovakia), $10 \mathrm{~mL}$ and $20 \mathrm{~mL}$ Luer lock syringes were purchased from B. Braun (Melsungen, Germany), and syringe combi-stoppers were also bought from B. Braun (Melsungen, Germany). The cellulose fiber sample pads (cat. no CFSP203000) were bought from Sigma-Aldrich (St. Louis, MO, USA). The BOLA PTFE sheets with a thickness of $0.05 \mathrm{~mm}$ were bought from Bohlender GmbH (Grünsfeld, Germany).

\section{D printing}

All the structures were created using Autodesk Fusion 360 software (San Rafael, CA, USA) following the conversion to ".stl" files by Autodesk Meshmixer software (San Rafael, CA, USA). The ".stl" files can be found in the Additional file 2. The ".stl" files were sliced by KISSlicer software v. 1.5 (http://www.kisslicer.com/) and transferred as G-codes to the 3D printer. The TRILAB DeltiX Mini FDM 3D printer using the delta kinematics printing (TriLAB Group, Brno, Czech Republic) was used for printing the structures. Filaments with a diameter of $1.75 \mathrm{~mm}$ contained PLA or ASA (Fillamentum Manufacturing Czech, Hulín, Czech Republic) and BVOH (Verbatim, Tokyo, Japan). All the printed structures were baked for $12 \mathrm{~h}$ at $60^{\circ} \mathrm{C}$ to prevent inhibitory effects on PDMS. 


\section{PDMS-based lab-in-a-syringe}

The PDMS-based lab-in-a-syringe devices were prepared as follows: the 3D printed structures were inserted into the syringe barrel and placed in the correct position by common modelling clay (bought from the local supermarket). The syringe tip was also blocked by the modelling clay. The syringe barrel was then placed vertically ("on the syringe tip") and PDMS was poured into the barrel. The PDMS was prepared beforehand by mixing the prepolymer and the curing agent (Sylgard 184) in a ratio of 10:1, stirred properly, and degassed in a vacuum for $15 \mathrm{~min}$. The amount of PDMS poured into the barrel depended on the structure of the LIS device (small parts of 3D printed structures should stay above the PDMS to allow their mechanical removal). Then the barrel containing PDMS and 3D-printed structures was inserted into the desiccator under vacuum and kept there for at least $48 \mathrm{~h}$ at room temperature $\left(\sim 25^{\circ} \mathrm{C}\right)$. Subsequently, the modelling clay parts were removed, and the $3 \mathrm{D}$ printed parts were also very carefully (mechanically) removed using common tweezers. The channels were then carefully rinsed with deionized water.

In the case of preparing "two-step" PDMS-based LIS devices, the $\mathrm{KBr}$ structures (prepared via the application of pressure by the $\mathrm{KBr}$ tool kit for IR spectroscopy) as well as the novel $3 \mathrm{D}$ printed structures were placed into the right position by using formed channels as well as modelling clay. The PDMS was then poured into the syringe barrel; the volume of PDMS again depended on the structure of the LIS device. Finally, the syringe barrel was closed via the plunger (with a PTFE sheet on it) as close as possible to the second 3D printed channel (The closing was also regulated by the movement of the second $3 \mathrm{D}$ printed channel through the syringe tip because it regulates pressure differences.) and placed vertically ("on the plunger"). This caused the flow of PDMS from the open parts made in the first step to the right positions for curing in the second step. Then the syringe was placed into the desiccator under vacuum and kept for at least $48 \mathrm{~h}$ at room temperature $\left(\sim 25^{\circ} \mathrm{C}\right)$. After that, the syringe plunger was carefully removed. The modelling clay parts as well as the 3D printed parts were also very carefully removed. The final channels were rinsed with deionized water, dried, and the LIS device was closed using the plunger and the stopper (at the tip side).

\section{Protein determination assay}

The commercial Bradford reagent (cat no. B6916) from Sigma-Aldrich (St. Louis, MO, USA) was used to quantify the total protein content. The procedure is based on the formation of a complex between the dye, Brilliant Blue G, and the proteins in the solution. The protein-dye complex causes a shift in the dye's absorption maximum from $465 \mathrm{~nm}$ (yellowish green) to $595 \mathrm{~nm}$ (blue). The commercial reagent $(0.5 \mathrm{~mL})$ was directly injected via a syringe with a needle into the reservoir in the LIS device. The device was closed by the stopper and kept in the refrigerator. Prior to use, the LIS device was brought to room temperature and gently shaken. The syringe was then filled with sample by moving the plunger in the syringe two marks (representing approximately $6.5 \mathrm{~mm}$ ). The LIS device was closed with the stopper, gently shaken, and incubated for five minutes at room temperature. The color intensity was analyzed by taking photos of the device using a common smartphone (13 mpx camera), which were then transferred to Corel Photo-Paint X4 software. The mask tool was used to pick the colored region, and the histogram was analyzed. The average value with the standard deviation value was used for calibration (the color intensity was calculated as 255 minus the average value, as described previously (Hárendarčíková et al. 2017)).

\section{Results and discussion}

The aim of this work was to develop a low-cost, portable lab-in-a-syringe device for the fast and easy determination of total protein content. There are two issues that need to be solved: (i) the volume of the syringe is quite big for the sampling of biopharmaceuticals, (ii) the Bradford reagent, as the "standard" assay, cannot be used with the cellulose fiber pad. When the reagent was placed on the pad, it formed a blue-colored product. This was probably due to the use of proteins in the production process of paper-based materials. The same was observed for other paper-based materials (including Whatman filter papers).

This led us to the idea of developing "second generation" lab-in-a-syringe devices. The idea was to form a reaction container in the syringe barrel where the Bradford reagent can be stored. The design should also pick up the sample without contaminating the sample with the reagent. Generally, this concept can be used for other reagents and used e.g., in terrain analysis. In our work, we proposed two types of single-use LIS devices (see Fig. 1). The first contains only the reservoir for the reagent and the sampling channel while the second also contains fluidic channels for the volume minimization.

Theoretically, the first device with the reservoir only can be used repeatedly. However, the cleaning procedure should be very efficient to be acceptable for the analytical purposes. Hence, we proposed these devices as the single-use ones. The preparation of both devices started with the 3D printing of reservoir structures and sampling channels (Fig. 2a). They were inserted into the syringe barrel and PDMS was poured (Fig. 2b, c). The structures were then mechanically removed (Fig. 2d). In the case of 


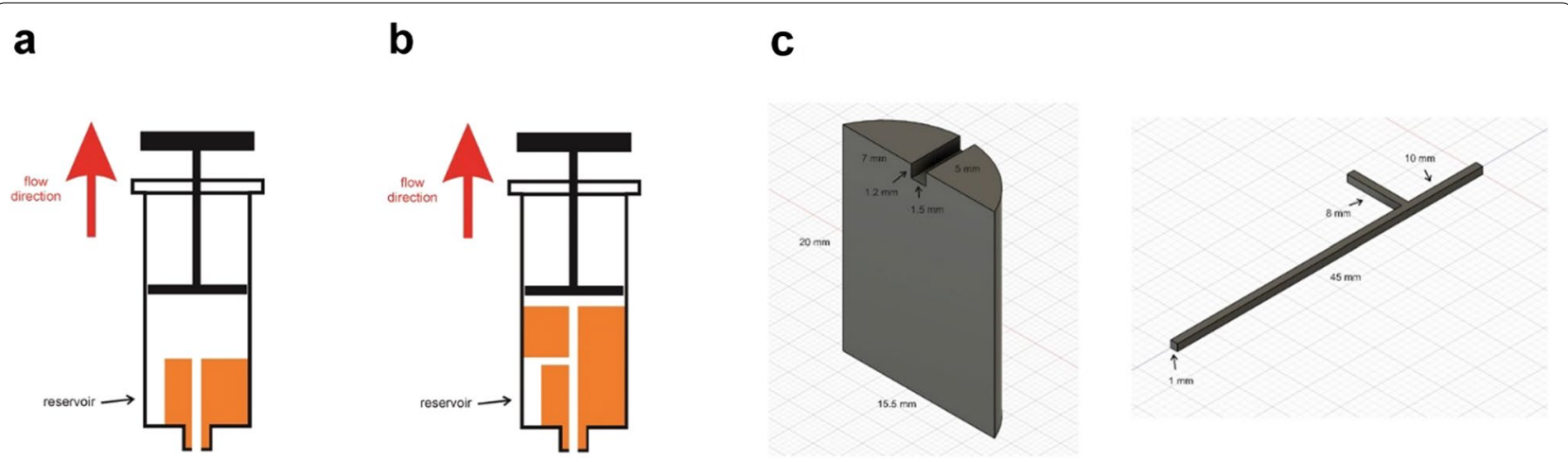

Fig. 1 Scheme of the "second generation" (reservoir-based) lab-in-a-syringe devices. a LIS device with a reservoir; $\mathbf{b}$ LIS device with the reservoir and fluidic channels; $\mathbf{c}$ dimensions of the reservoir and the channel

the second design, a second step in production was performed. Again, the channel structures were placed in the right positions and PDMS was poured into the reversed position of the syringe barrel (see Fig. 2e-i). The photos of the preparation process are in Fig. S1 in the Additional file 1.
Interestingly, the most problematic step was keeping the channel connecting the reaction reservoir with the main straight channel open. The PDMS is too viscous and usually clogs the channel (or the channel was not formed) when there is no filling during curing. To prevent such issues, a barrier should be used. However, a

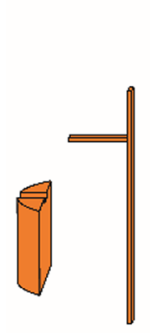

3D printed structures b

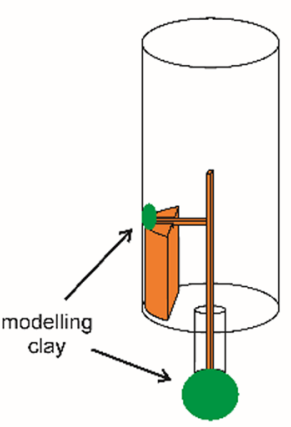

C

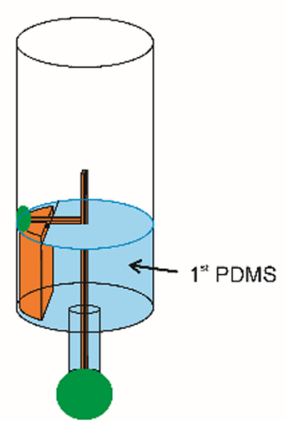

h

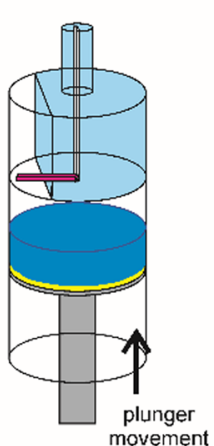

d

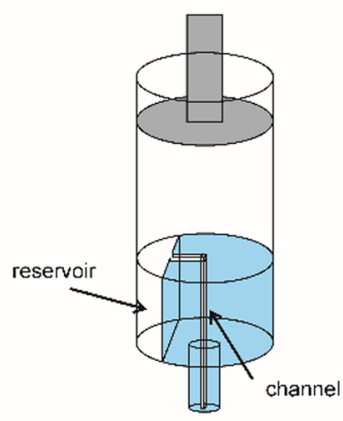

i
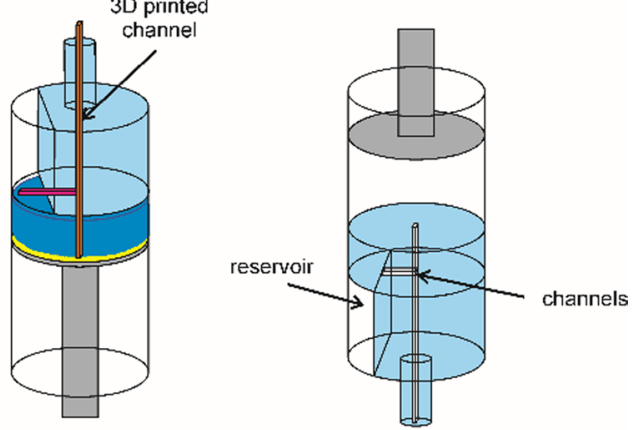

Fig. 2 Scheme of the preparation of the LIS devices 
when using 3D printed structures, it is not possible to mechanically remove them (they are orthogonal to the walls of the syringe barrel). Therefore, we studied two different setups: (i) the use of pressed $\mathrm{KBr}$ structures that could be dissolved in water after PDMS curing, and (ii) the use of water-soluble $\mathrm{BVOH}$ filaments to $3 \mathrm{D}$ print this structure. The first method showed promise, but the small $\mathrm{KBr}$ structures were very fragile and manipulation before PDMS curing was not easy (KBr structures should fit PDMS cured in the first step perfectly). However, the solubility of $\mathrm{KBr}$ in water was rapid. After PDMS curing, the LIS device was submerged in a hot water bath $\left(40{ }^{\circ} \mathrm{C}\right)$, and the channels were rinsed with hot water. In the second method, insertion of the BVOH part into the syringe barrel was very easy, but this part takes longer to dissolve; it took more than four hours to dissolve the $\mathrm{BVOH}$ structure in nearly boiling water. Moreover, it is necessary to minimize the contact of boiling water with the syringe material as it is not thermostable. The $\mathrm{BVOH}$ part could be dissolved with water at laboratory temperature for more than three days. The BVOH was used for further studies mainly because of the easier manipulation in comparison with $\mathrm{KBr}$. After the preparation of the LIS device, it was flushed with deionized water and dried under room temperature overnight. The final device was then filled with the Bradford reagent (which does not react with any component of the device), closed by the stopper, and kept in the fridge for further use (Fig. 3a). The use of these devices is representatively depicted in Fig. 3b, c, for both LIS designs.

Then the repeatability of the preparation was studied on ten LIS devices (both with the reservoir and with the reservoir and fluidic channels). First, deviations in both reservoir and channel dimensions were evaluated.
The height and the width of the reservoir differed maximally in $5 \%$ and $4 \%$. The width of the channel varied in maximally $10 \%$. The worst parameter is the width of the channel connecting the reaction reservoir with the main straight channel in the case of the second design (Fig. 1b) where the difference is about $35 \%$. Hence, the first design (Fig. 1a) was chosen for the next analyses. The stability of the reagent in the LIS device was further investigated. 20 devices were prepared, filled with the Bradford reagent, and stored in the fridge (at $4{ }^{\circ} \mathrm{C}$ ). Each day; $200 \mu \mathrm{g} \mathrm{mL}^{-1}$ BSA solution was analyzed by the LIS device. Repeatability of the color intensity was calculated to be $96 \%$ that is acceptable in the view of the stability of the reagent (Fig. S2 in the Additional file 1). Next, the portability (utilization of the device in a terrain) was studied. LIS devices were taken (after preparation and one-day storage in a fridge) in a car under a sunshine in an insulated cooler bag for $6 \mathrm{~h}$. The temperature in the cooler bag increased from 4 to $15^{\circ} \mathrm{C}$. Then, the LIS devices $(N=5)$ were taken back to the room temperature and BSA solution $\left(200 \mu \mathrm{g} \mathrm{mL}^{-1}\right)$ was analyzed and the color intensity was compared to those keeping in the fridge. The color intensity measured by the LIS devices stored in a car was not less than 93\% than that stored in the fridge. This seems to be very good for the low-cost portable assay. The Bradford assay can generally suffer from interferences caused by e.g., carbohydrates or lipids. However, the interferences will be the same for the assay in a LIS device as well as in a flask. Moreover, the biopharmaceutical samples of interest contain mainly proteins, so we did not suppose any interference in our samples.

Finally, the developed lab-in-a-syringe device was applied to determine total protein content in

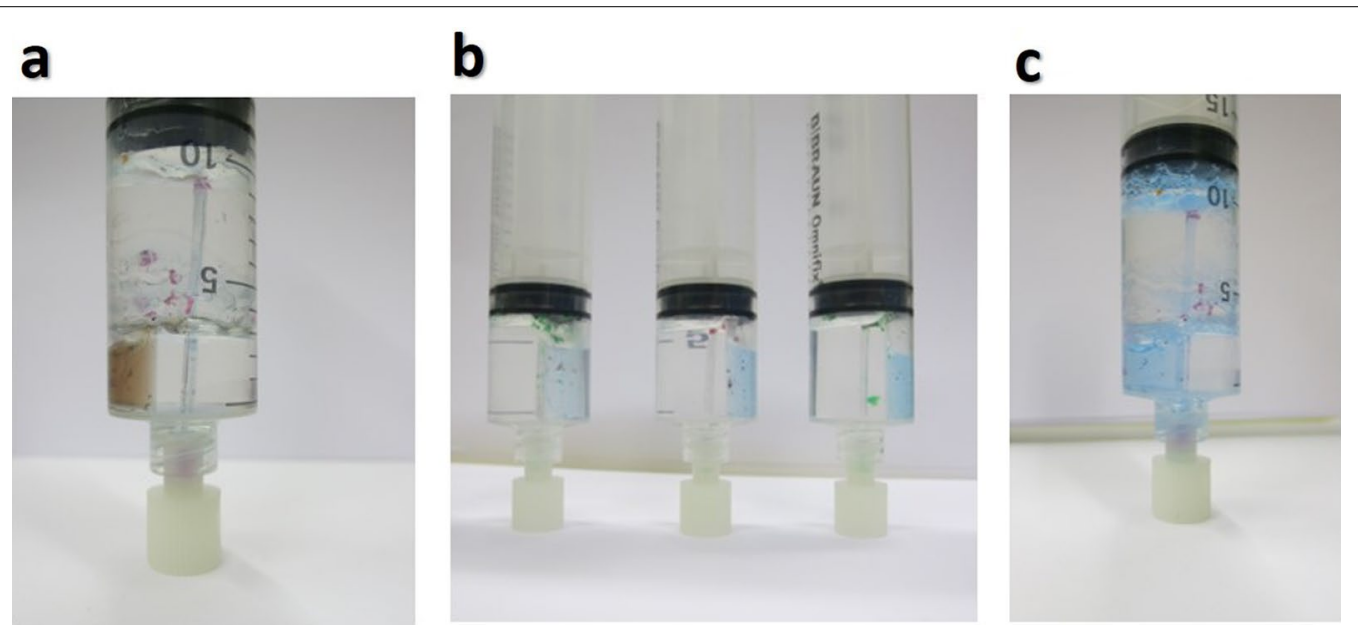

Fig. 3 PDMS-assisted lab-in-a-syringe device for determination of total protein content. a Filled with the Bradford reagent; $\mathbf{b}$ after the reaction with BSA at the single PDMS curing device; $\mathbf{c}$ After the reaction with BSA at the two-step PDMS curing device 
biopharmaceutical samples. These samples were essentially cultivating media representing a huge mixture of proteins. Therefore, it was a profitable method to test the device's ability quickly and easily determine total protein content during production. The samples were provided by PrimeCell Advanced Therapy. First, we performed calibration measurements with our lab-in-a-syringe devices using BSA as the model sample in the concentration range of $60-1000 \mu \mathrm{g} \mathrm{mL}^{-1}$ (Fig. S3 in the Additional file 1). As can be seen, the calibration started to bend, as is typical for low-cost microfluidics (Martinez et al. 2007). However, the concentrations can be estimated by the linear equation in the range of $60-300 \mu \mathrm{g} \mathrm{mL}^{-1}$ and correlation coefficient of $0.9837\left(R^{2} 0.9676\right)$. In the end, we performed measurements on the biopharmaceutical samples by using the final LIS design. The samples were diluted 10times using a phosphate buffer $\mathrm{pH} 7.4$ to fit the calibration curve. The concentration observed was $1830 \pm 240 \mu \mathrm{g} \mathrm{mL}^{-1}(N=10)$. It was confirmed using the standard Bradford assay in a flask. There was not any difference observed at the 0.05 level of significance (determined by the t-test).

\section{Conclusions}

In our work, we developed a novel, low-cost, portable lab-in-a-syringe assay for determination of total protein content in biomedical and biopharmaceutical products. The device is based on the formation of 3D printed channels in PDMS in the syringe barrel. Moreover, we proposed, for the first time, a two-step fabrication procedure to produce more complicated devices that allow storage of the reagent, too. However, the use of easier design is more profitable from the practical point of view. It allows storing the reagent and it is more precise in the determination part of the assay. Generally, we believe that the production procedures could be used in the future to prepare of other devices for more complicated analytical tasks. Also, there will be new materials for 3D printing (as e.g. AquaSys materials) that will probably allow to dissolve the connection parts (barriers) more quickly than when using $\mathrm{BVOH}$.

\section{Supplementary Information}

The online version contains supplementary material available at https://doi. org/10.1186/s40543-021-00307-0.

Additional file 1. Figure S1: Preparation of PDMS-based LIS devices. Figure S2: Stability of the reagent in the LIS device. Figure S3: Example of the calibration curve.

Additional file 2. LIS_channel.stl. Drawing of the straight channel used for 3D printing. LIS_Tchannel.stl. Drawing of the T-channel used for 3D printing. LIS_reservoir.stl. Drawing of the reservoir used for 3D printing.
Acknowledgements

Not applicable.

\section{Authors' contributions}

Investigation: ED, KK, AŠ, DB, PŠ; Data curation: KV, VP; Methodology: JP, Conceptualization: JP; Funding acquisition: LP, JP; Resources: SF, LP; Supervision: ZK, SF, JP; Writing: JP. All the authors read and approved the final manuscript.

\section{Funding}

This work was supported by the Ministry of Industry and Trade of the Czech Republic (project CZ.01.1.02/0.0/0.0/16_084/0010317), the Palacký University Olomouc (project IGA_PrF_2021_021), and the Ministry of Education, Youth and Sports of the Czech Republic (Project CZ.02.1.01/0.0/0.0/16_19/0000754).

\section{Availability of data and materials}

The data and materials are available from the corresponding author on reasonable request.

\section{Declaration}

\section{Competing interests}

The authors declare that they have no competing interests.

\section{Author details}

${ }^{1}$ Department of Analytical Chemistry, Faculty of Science, Palacký University Olomouc, 17. Listopadu 12, 77146 Olomouc, Czech Republic. ${ }^{2}$ Primecell Advanced Therapy, Jáchymova 26/2, 11000 Praque, Czech Republic.

Received: 11 May 2021 Accepted: 3 November 2021

Published online: 25 November 2021

\section{References}

Akyazi T, Basabe-Desmonts I, Benito-Lopez F. Review on microfluidic paperbased analytical devices towards commercialisation. Anal Chim Acta. 2018;1001:1-17.

Au AK, Huynh W, Horowitz LF, Folch A. 3D-printed microfluidics. Angew Chem Int Ed. 2016;55:3862-81.

Barreto JA, de Assis RD, Cassella RJ, Lemos VA. A novel strategy based on insyringe dispersive liquid-liquid microextraction for the determination of nickel in chocolate samples. Talanta. 2019;193:23-8.

Beauchamp MJ, Nielsen AV, Gong H, Nordin GP, Woolley AT. 3D printed microfluidic devices for microchip electrophoresis of preterm birth biomarkers. Anal Chem. 2019;91:7418-25.

Bhattacharjee N, Urrios A, Kang S, Folch A. The upcoming 3D-printing revolution in microfluidics. Lab Chip. 2016;16:1720-42.

Bhattacharjee N, Parra-Cabrera C, Kim YT, Kuo AP, Folch A. Desktopsterolitography 3D-printing of a poly(dimethylsiloxane)-based material with Sylgard-184 properties. Adv Mater. 2018;30:1800001.

Bradford MM. A rapid and sensitive method for the quantitation of microgram quantities of protein utilizing the principle of protein-dye binding. Anal Biochem. 1976;72:248-54.

Cankařová N, La Venia A, Krajčovičová S, Krchňák V. Configuration-dependent medium-sized ring formation: chiral molecular framework with three-dimensional architecture. J Org Chem. 2019;84:636-44.

Carrell C, Kava A, Nguyen N, Menger R, Munshi Z, Call Z, Nussbaum N, Henry C. Beyond the lateral flow assay: a review of paper-based microfluidics. Microelectron Engineer. 2019;206:45-54.

Dahlberg T, Stangner T, Zhang H, Wiklund K, Lundberg P, Edman L, Andersson M. 3D printed water-soluble scaffolds for rapid production of PDMS micro-fluidic flow chambers. Sci Rep. 2018:8:3372.

Dixit CK, Kadimisetty K, Rusling J. 3D-printed miniaturized fluidic tools in chemistry and biology. Trend Anal Chem. 2018;106:37-52.

Gong H, Woolley AT, Nordin GP. 3D printed high density, reversible chip-tochip microfluidic interconnects. Lab Chip. 2018;18:639-47.

Hárendarčíková L, Baron D, Šebestová A, Rozsypal J, Petr J. True lab-in-asyringe technology for bioassays. Talanta. 2017;174:285-8.

Hartings MR, Ahmed Z. Chemistry from 3D printed objects. Nature Rev Chem. 2019:3:305-14. 
Hidalgo D, Sanchez R, Lalaleo L, Bonfill M, Corchete P, Palazon J. Biotechnological production of pharmaceuticals and biopharmaceuticals in plant cell and organ cultures. Current Med Chem. 2018;25:3577-96.

Horstkotte B, Atochero NLD, Solich P. Lab-in-syringe automation of stirringassisted room-temperature headspace extraction coupled online to gas chromatography with flame ionization detection for determination of benzene, toluene, ethylbenzene, and xylenes in surface waters. J Chromatogr A. 2018;1555:1-9.

Hwang Y, Paydar OH, Candler RN. 3D printed molds for non-planar PDMS microfluidic channels. Sens Actuators A. 2015;226:137-42.

Kamali B, Asiaei S, Beigzadeh B, Ebadi AA. Micro-lithography on paper, surface process modifications for biomedical performance enhancement. Colloid Surf A. 2018:555:389-96.

Li F, Smejkal P, Macdonald NP, Guijt RM, Breadmore MC. One-step fabrication of a microfluidic device with an integrated membrane and embedded reagents by multimaterial 3D printing. Anal Chem. 2017a;89:4701-7.

Li F, Macdonald NP, Guijt RM, Breadmore MC. Using printing orientation for tuning fluidic behavior in microfluidic chips made by fused deposition modeling 3D printing. Anal Chem. 2017b;89:12805-11.

Li F, Macdonald NP, Guijt RM, Breadmore MC. Increasing the functionalities of 3D printed microchemical devices by single material, multimaterial, and print-pause-print 3D printing. Lab Chip. 2019a;19:35-49.

Li F, Macdonald NP, Guijt RM, Breadmore MC. Multimaterial 3D printed fluidic device for measuring pharmaceuticals in biological fluids. Anal Chem. 2019b;91:1758-63.

Lomonossoff GP, D'Aoust MA. Plant-produced biopharmaceuticals: a case of technical developments driving clinical deployment. Science. 2016;353:1237-40.

Macdonald NP, Cabot JM, Smejkal P, Guijt R, Paull B, Breadmore MC. Comparing microfluidic performance of three-dimensional (3D) printing platforms. Anal Chem. 2017:89:3858-66.

Manzanares Palenzuela CL, Pumera M. (Bio)Analytical chemistry enabled by 3D printing: Sensors and biosensors. Trends Anal Chem. 2018;103:110-8.

Martinez AW, Phillips ST, Butte MJ, Whitesides GM. Patterned paper as a platform for inexpensive, low-volume, portable bioassays. Angew Chem Int Ed. 2007:46:1318-20.

Maskova T, Harendarcikova L, Petr J. Determination of Escherichia coli in urine using a low-cost foil-based microfluidic device. Talanta. 2017:170:36-40.

Maya F, Horstkotte B, Estela JM, Cerda V. Lab in a syringe: fully automated dispersive liquid-liquid microextraction with integrated spectrophotometric detection. Anal Bioanal Chem. 2012;404:909-17.

Mikhail IE, Tehranirokh M, Gooley AA, Guijt RM, Breadmore MC. In-syringe electrokinetic focusing of amphoteric analytes for direct injection electrospray ionization mass spectrometry. Anal Chem. 2019:91:8259-66.

Montgomery RH, Phelan K, Stone SD, Decuir F, Hollins BC. Photolithographyfree PDMS stamps for paper microdevice fabrication. Rapid Prototyp J. 2018;24:361-7.

Parr MK, Montacir O, Montacir H. Physicochemical characterization of biopharmaceuticals. J Pharm Biomed Anal. 2016;130:366-89.

Pauli GEN, de la Escosura-Muniz A, Parolo C, Bechtold IH, Merkoci A. Lab-ina-syringe using gold nanoparticles for rapid immunosensing of protein biomarkers. Lab Chip. 2015;15:399-405.
Radosevich M, Burnouf T. Intravenous immunoglobulin G: trends in production methods, quality control and quality assurance. Vox Sang. 2010:98:12-28.

Raynal B, Lenormand P, Baron B, Hoos S, England P. Quality assessment and optimization of purified protein samples: why and how? Microb Cell Factor. 2014;13:180.

Romanov V, Samuel R, Chaharlang M, Jafek AR, Frost A, Gale BK. FDM 3D printing of high-pressure, heat-resistant, transparent microfluidic devices. Anal Chem. 2018:90:10450-6.

Saggiomo V, Velders AH. Simple 3D printed scaffolds-removal method for the fabrication of intricate microfluidic devices. Adv Sci. 2015;2:1500125.

Salentijn Gl, Oomen PE, Grajewski M, Verpoorte E. Fused deposition modeling $3 \mathrm{D}$ printing for (Bio)analytical device fabrication: procedures, materials, and applications. Anal Chem. 2017:89:7053-61.

Salentijn GIJ, Grajewski M, Verpoorte E. Reinventing (bio)chemical analysis with paper. Anal Chem. 2018;90:13815-25.

Schütznerová E, Oliver AG, Přibylka A, Krchňák V. Solid-phase synthesis of tetramic acid via resin-bound enol ethers as a privileged scaffold in drug discovery. Adv Synth Catal. 2018;360:3693-9.

Šrámková I, Horstkotte B, Fikarová K, Sklenářová H, Solich P. Direct-immersion single-drop mixtroextraction and in-drop stirring microextraction for the determination of nanomolar concentrations of lead using automated lab-in-syringe technique. Talanta. 2018;184:162-72.

Tang S, Sun J, Xia D, Zang B, Gao Y, Chen C, Shen W, Lee HK. In-syringe extraction using compressible and self-recoverable, amphiphilic graphene aerogel as sorbent for determination of phenols. Talanta. 2019;195:165-72.

Torano JS, Ramautar R, de Jong G. Advances in capillary electrophoresis for the life sciences. J Chromatogr B. 2019;1118:116-36.

Vittayarukskul K, Lee AP. A truly Lego ${ }^{\circledR}$-like modular microfluidics platform. J Micromech Microeng. 2017;27:035004.

Waheed S, Cabot JM, Macdonald NP, Lewis T, Guijt RM, Paull B, Breadmore MC. 3D printed microfluidic devices: enablers and barriers. Lab Chip. 2016;16:1993-2013.

Weng X, Kang YJ, Guo Q, Peng B, Jiang H. Recent advances in threadbased microfluidics for diagnostic applications. Biosens Bioelectron. 2019;132:171-85

Yu Y, You J, Sun Z, Li G, Ji Z, Zhang S, Zhou X. Determination of residual organophosphorus thioester pesticides in agricultural products by chemical isotope-labelling liquid chromatography-tandem mass spectrometry coupled with in-syringe dispersive solid phase clean-up and in situ cleavage. Anal Chim Acta. 2019;1055:44-55.

Zor E, Bekar N. Lab-in-a-syringe using gold nanoparticles for rapid colorimetric chiral discrimination of enantiomers. Biosens Bioelectron. 2017;91:211-6.

\section{Publisher's Note}

Springer Nature remains neutral with regard to jurisdictional claims in published maps and institutional affiliations.

\section{Submit your manuscript to a SpringerOpen ${ }^{\circ}$ journal and benefit from:}

- Convenient online submission

- Rigorous peer review

- Open access: articles freely available online

- High visibility within the field

- Retaining the copyright to your article

Submit your next manuscript at springeropen.com 